\title{
Nonperturbative Heavy-Quark Diffusion in the Quark-Gluon Plasma
}

\author{
H. van Hees ${ }^{1}$, M. Mannarelli ${ }^{2}$, V.Greco ${ }^{3}$ and R. Rapp ${ }^{1}$ \\ ${ }^{1}$ Cyclotron Institute and Physics Department, Texas AEMM University, College Station, Texas 77843-3366, U.S.A. \\ ${ }^{2}$ Instituto de Ciencias del Espacio (IEEC/CSIC), E-08193 Bellaterra (Barcelona), Spain and \\ ${ }^{3}$ Dipartimento di Fisica e Astronomia, Via S. Sofia 64, I-95125 Catania, Italy
}

(Dated: October 31, 2018)

\begin{abstract}
We evaluate heavy-quark (HQ) transport properties in a Quark-Gluon Plasma (QGP) within a Brueckner many-body scheme employing interaction potentials extracted from thermal lattice QCD. The in-medium $T$-matrices for elastic charm- and bottom-quark scattering off light quarks in the QGP are dominated by attractive meson and diquark channels which support resonance states up to temperatures of $\sim 1.5 T_{c}$. The resulting drag coefficient increases with decreasing temperature, contrary to expectations based on perturbative QCD scattering. Employing relativistic Langevin simulations we compute HQ spectra and elliptic flow in $\sqrt{s_{N N}}=200 \mathrm{GeV}$ Au-Au collisions. A good agreement with electron decay data supports our nonperturbative computation of HQ diffusion, indicative for a strongly coupled QGP.
\end{abstract}

PACS numbers: 25.75.-q 25.75.Dw 25.75.Nq

Experiments at the Relativistic Heavy-Ion Collider (RHIC) have shown that the matter produced in $\mathrm{Au}-\mathrm{Au}$ collisions cannot be described by a weakly interacting gas of quarks and gluons, but rather consists of a strongly coupled Quark-Gluon Plasma (sQGP) with remarkably large opacity and low viscosity. The latter is required by hydrodynamic descriptions of the expanding fireball, implying rapid thermalization of the medium [1, 2]. The understanding of these properties in terms of the underlying interactions in the QGP, as governed by Quantum Chromodynamics (QCD), is a key theoretical objective. A valuable probe of the sQGP are heavy quarks (charm and bottom) which, due to their large mass, $m_{Q} \gg T_{c}$ $\left(T_{c} \simeq 180 \mathrm{MeV}\right.$ : critical temperature [3]), are believed to be sensitive to the processes that establish and maintain thermalization of the medium, even at soft momentum scales. RHIC data for single-electron $\left(e^{ \pm}\right)$spectra associated with semileptonic heavy-quark (HQ) decays in $\mathrm{Au}-\mathrm{Au}$ collisions exhibit a surprisingly strong suppression and elliptic flow [4, 5, 6], indicating substantial collective behavior of charm quarks in the expanding fireball. Perturbative QCD (pQCD) calculations, based on radiative energy loss, cannot explain these findings, even after inclusion of elastic scattering 7, 8]. Furthermore, it has been argued that the convergence of the perturbative series for the HQ diffusion constant is rather poor [9], which calls for nonperturbative approaches. Effective models with strong HQ coupling in the QGP [10, 11, 12, 13] lead to significantly reduced thermal relaxation times compared to pQCD elastic scattering [14], resulting in better agreement [15, 16] with $e^{ \pm}$spectra [4, 5, 6] .

In the present article, we perform a microscopic calculation of HQ diffusion in the QGP employing a nonperturbative $T$-matrix approach [17] with a driving kernel (potential) estimated from finite-temperature lattice QCD computations. We include a complete set of color channels for heavy-light quark interactions, as well as $l=0,1$ partial waves together with HQ spin symmetry. This, in principle, provides an estimate of (elastic) transport coefficients without tunable parameters, albeit significant uncertainties remain in the definition of the potential. Within these uncertainties applications to HQ observables at RHIC support our approach.

To evaluate in-medium properties of heavy quarks $(Q=c, b)$ and heavy-light quark correlations, we employ a Brueckner-type many-body approach [17]. Our key assumptions are: (i) the main features of the elastic heavylight quark interaction can be approximated by a static interaction potential, $V(r)$ (to leading order in $1 / m_{Q}$, such an approach has been successfully applied for $D$ meson spectra and decays in the vacuum [18, 19]), and (ii) $V(r)$ can be extracted from lQCD simulations of the singlet-free energy $F_{1}(r, T)[20]$ for a static $\bar{Q} Q$ pair. As in previous works [17, 21, 22, 23], we identify the potential with the internal energy, $U_{1}$, which is obtained by subtracting the entropy contribution from the free energy,

$$
V_{1}(r, T)=U_{1}(r, T)-U_{1}(\infty, T), \quad U_{1}=F_{1}-T \frac{\mathrm{d} F_{1}}{\mathrm{~d} T}
$$

A further subtraction is required to ensure the vanishing of the potential at large distance and thus the convergence of the $T$-matrix integral equation. In lQCD simulations the large distance limit of the internal energy, $U_{1}\left(\infty, T>T_{c}\right)$, is a decreasing function of the temperature. It is tempting to associate this quantity with a selfenergy contribution to the HQ mass, $m_{Q}(T)=m_{0}+$ $U_{1}(\infty, T) / 2\left(m_{0}\right.$ : "bare" mass). However, around $T_{c}$, $U_{1}(\infty, T)$ develops a rather pronounced maximum structure rendering a mass interpretation problematic. Furthermore, little is known about the momentum dependence of this quantity. For simplicity, we assume constant 
values for effective $c$ - and $b$-quark masses of $m_{c}=1.5 \mathrm{GeV}$ and $m_{b}=4.5 \mathrm{GeV}$ (the difference to the current mass is mainly attributed to perturbative contributions).

In addition to the color-singlet (meson) channel, we consider HQ interactions in the color-octet $Q \bar{q}$, as well as in antitriplet and sextet $Q q$ (diquark) channels. For the corresponding potentials we adopt Casimir scaling according to leading-order $(\mathrm{LO})$ perturbation theory, $V_{8}=$ $-\frac{1}{8} V_{1}, V_{\overline{3}}=\frac{1}{2} V_{1}, V_{6}=-\frac{1}{4} V_{1}$, which is also supported by lQCD calculations of the finite- $T$ HQ free energy [24, 25].

The largest uncertainty in our calculations resides in the definition and extraction of the potential. While the identification with the internal energy (rather than the free energy) may be considered as an upper limit, the variations between different 1 QCD calculations and pertinent parametrizations to numerically evaluate the entropy term in Eq. (1), are appreciable. We have adopted 3 different potentials, based on parametrizations of $F_{1}(r, T)$ in Refs. [22] $=[\mathrm{Wo}],[21]=[\mathrm{SZ}]$ and $[17]=[\mathrm{MR}]$ for quenched [20], 2-flavor [26] and 3-flavor lQCD [27], respectively. The parametrizations [Wo] and [SZ] are similar to a recent extraction [23] from 3-flavor lQCD [28]. The $[\mathrm{MR}]$ potential is deeper than the other two for $T \lesssim 1.6 T_{c}$ (and consequently gives larger effects), but falls off faster above. The resulting transport coefficients vary by $\sim 40 \%$. More details will be elaborated in an extended paper [29]; in the following we restrict ourselves to the [Wo] potential. The lQCD-based potentials are implemented into a Brueckner many-body approach for heavy quarks, defined by a system of coupled Bethe-Salpeter (BS) and Dyson equations:

$$
\begin{aligned}
T & =K+\int K G T \\
\Sigma^{Q} & =\Sigma_{g}+\int T S^{q}, \quad S^{Q}=S_{0}^{Q}+S_{0}^{Q} \Sigma^{Q} S^{Q},
\end{aligned}
$$

with $T$ : heavy-light quark $T$-matrix, $K$ : interaction kernel, $G$ : 2-particle propagator, $S^{Q, q}\left(S_{0}^{Q, q}\right)$ : (free) singleparticle propagators for heavy and light quarks, $\Sigma^{Q}$ : HQ selfenergy with contributions from thermal gluons $\left(\Sigma_{g}\right)$ and the $T$-matrix part from interactions with thermal light-anti-/quarks. Since we focus on a QGP at zero chemical potential $\left(\mu_{q}=0\right)$, all quantities are quarkantiquark symmetric. To close the equations in the quark sector, one needs the corresponding system of equations for the light sector, which has been solved selfconsistently for $\Sigma_{q}$ and $T_{q \bar{q}}$ in Ref. [17]. Here, we augment these results by $q q$ diquark interactions and implement the quark selfenergies in simplified form with constant real and imaginary parts as an effective quark mass, $m_{q}=0.25 \mathrm{GeV}$, and width, $\Gamma_{q}=0.2 \mathrm{GeV}$ [17]; variations in these parameters have little impact on the resulting quark selfenergies. The effects from heavy quarks in the heat bath can be safely neglected.

To implement the (static) 1QCD-based potential into our Brueckner approach we adopt the following approximations. First, we use a standard reduction scheme 30]

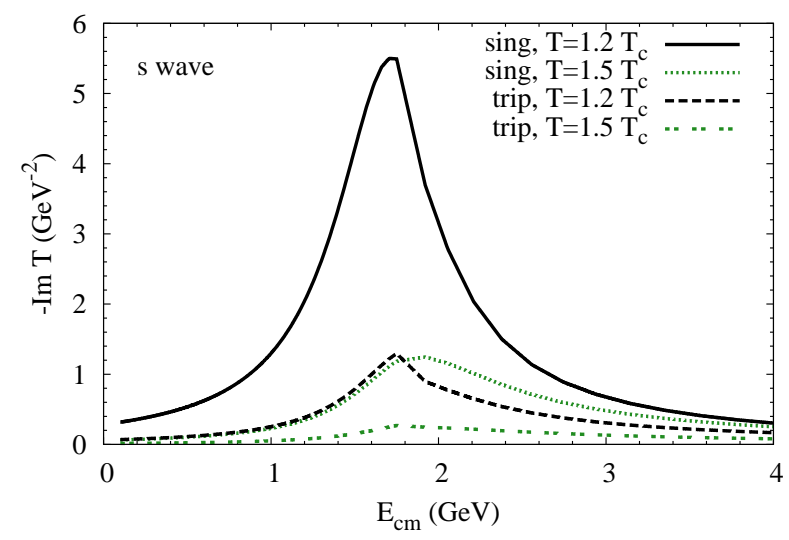

FIG. 1: Imaginary part of the in-medium $T$-matrix for $S$-wave $c \bar{q}$ and $c q$ scattering in color-singlet and antitriplet channels, respectively, at two different temperatures. The two-body threshold is at $E_{\mathrm{thr}}=m_{c}+m_{q}=1.75 \mathrm{GeV}$.

to convert the 4-dimensional BS equation into a 3D Lippmann-Schwinger (LS) equation (other reductions [31] lead to very similar results 17]), thus neglecting virtual particle-antiparticle loops but keeping relativistic kinematics of the quark propagators. This, in turn, enables to identify the reduced kernel $K$ with the potentials $V_{1, \overline{3}, 6,8}$ constructed above, representing a ladder approximation to the $T$-matrix. As in Refs. [17, 21], we correct the static potentials for a relativistic Breit (current-current) interaction. Azimuthal symmetry and a partial-wave expansion lead to a 1-D LS equation in each color $(a)$ and angular-momentum $(l)$ channel $(E$ : center-of-mass energy of the $Q q$ system),

$$
\begin{array}{r}
T_{a, l}\left(E ; q^{\prime}, q\right)=V_{a, l}\left(q^{\prime}, q\right)+\frac{2}{\pi} \int \mathrm{d} k k^{2} V_{a, l}\left(q^{\prime}, k\right) \\
\quad \times G_{Q q}(E ; k) T_{a, l}(E ; k, q)\left[1-f\left(\omega_{k}^{Q}\right)-f\left(\omega_{k}^{q}\right)\right] .
\end{array}
$$

$f(\omega)$ denote Fermi-Dirac distributions and $\omega_{k}^{i}=\left(m_{i}^{2}+\right.$ $\left.k^{2}\right)^{1 / 2}$ quasiparticle dispersion laws. We include both $S$ $(l=0)$ and $P$-wave $(l=1)$ channels. The 2 -particle propagator in the Thompson scheme [30] reads

$$
G(E ; k)=(1 / 4) /\left[E-\left(\omega_{k}^{q}+\mathrm{i} \Sigma_{I}^{q}\right)-\left(\omega_{k}^{Q}+\mathrm{i} \Sigma_{I}^{Q}\right)\right] .
$$

Results for the in-medium $S$-wave $T$-matrix are illustrated in Fig. 1 for $c$-quark scattering. The attractive color-singlet and -antitriplet channels are the dominant contributions, supporting resonance structures up to temperatures of $\sim 1.7 T_{c}$ and $\sim 1.4 T_{c}$, respectively. Both the repulsive color channels, as well as $P$-waves, lead to much smaller $T$-matrices. However, due to larger degeneracies their contribution to the HQ selfenergies and transport coefficients is not negligible.

Next we compute the HQ selfenergies and transport coefficients generated by the nonperturbative heavy-light $T$-matrices. The HQ selfenergy represented by the second term on the right-hand-side of Eq. (3) is calculated 


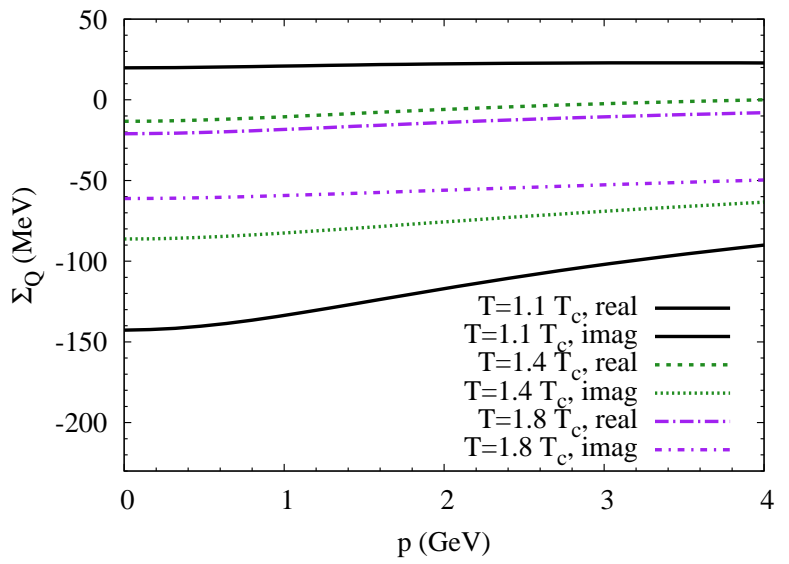

FIG. 2: Real (upper lines) and imaginary (lower lines) parts of the on-shell $c$-quark selfenergy versus 3 -momentum at temperatures $T=1.1,1.4$ and $1.8 T_{c}$.



FIG. 3: Friction coefficients of charm quarks vs. 3-momentum for different temperatures in a QGP, based on our nonperturbative $T$-matrix approach (upper curves at $p=0$ ) and on LO pQCD scattering with $\alpha_{s}=0.4$ (lower curves).

within the imaginary-time formalism as

$$
\begin{aligned}
\Sigma_{a}^{Q}\left(z_{v} ; p\right)= & \frac{d_{S I} d_{a}}{6} \int \frac{\mathrm{d}^{3} p^{\prime}}{(2 \pi)^{3}}(-T) \\
& \times \sum_{z_{\nu^{\prime}}} T_{a}\left(z_{\nu}+z_{\nu^{\prime}} ; \mathbf{p}, \mathbf{p}^{\prime}\right) D_{\bar{q}}\left(z_{\nu^{\prime}}, \mathbf{p}^{\prime}\right)
\end{aligned}
$$

$\left(z_{\nu}=\mathrm{i} \pi(2 \nu+1) T\right.$ : fermionic Matsubara frequencies). As implicit in our $T$-matrix (potential) we assume spin and light-flavor degeneracy of the heavy-light interaction (in line with the free $D$-meson spectrum 32]), yielding $d_{S I}=4(12) N_{f}$ for $S(P)$-waves $\left(N_{f}=2.5\right.$ to account for the smaller strange-quark density). The resulting $c$ quark selfenergies (Fig. 2) translate into large in-medium widths of around $\Gamma_{c}=-2 \operatorname{Im} \Sigma_{c} \simeq 200 \mathrm{MeV}$ (consistent with our input parameters). The dominant meson and diquark contributions are about equal, while the $P$-wave amounts to $\sim 40 \%$. The nonperturbative real parts are small.

We evaluate HQ diffusion in the QGP within a Fokker-
Planck equation for the distribution function, $f_{Q}$ [14],

$$
\frac{\partial f_{Q}}{\partial t}=\frac{\partial}{\partial p_{i}}\left(p_{i} \gamma f_{Q}\right)+\frac{\partial^{2}}{\partial p_{i} \partial p_{j}}\left(B_{i j} f_{Q}\right)
$$

with momentum and temperature dependent friction $(\gamma)$ and diffusion $\left(B_{i j}\right)$ coefficients. They are calculated as in Ref. [10] using the appropriate relation between the invariant amplitude $\mathcal{M}$ and our in-medium $T$-matrix,

$$
\begin{aligned}
& \sum|\mathcal{M}|^{2}=\frac{64 \pi}{s^{2}}\left(s-m_{q}^{2}+m_{Q}^{2}\right)^{2}\left(s-m_{Q}^{2}+m_{q}^{2}\right)^{2} \\
& \times N_{f} \sum_{a} d_{a}\left(\left|T_{a, l=0}(s)\right|^{2}+3\left|T_{a, l=1}(s) \cos \left(\theta_{\mathrm{cm}}\right)\right|^{2}\right) .
\end{aligned}
$$

The non-perturbative thermal relaxation rates reach up to $\gamma \simeq 1 /(7 \mathrm{fm} / \mathrm{c})$ at low momenta close to $T_{c}$, a factor of $\sim 4$ larger than elastic pQCD scattering (but comparable to the resonance model of Ref. [10]), cf. Fig. [3] In contrast to other calculations available thus far, the thermalization rate decreases with temperature, due to the dissolving resonances induced by the screening in the lQCD-based potentials. The increase in quark density is overcompensated by the loss of interaction strength. This has important consequences for HQ observables at RHIC, as discussed below. The $\cos ^{2}\left(\theta_{\mathrm{cm}}\right)$ factor in $|\mathcal{M}|^{2}$ reduces the $P$-wave contribution to $\gamma$ to $\sim 20 \%$ of the $S$-wave. Combining $T$-matrix and pQCD contributions, the spatial HQ-diffusion constant at $p=0$ amounts to $D_{H Q}=T /\left(m_{c} \gamma\right) \simeq 5 / 2 \pi T$ at $T=200 \mathrm{MeV}$, a factor of $\sim 4$ smaller than in pQCD, thus corroborating the notion of a strongly coupled QGP at temperatures up to 1.5-2 $T_{c}$.

The above calculated transport coefficients (from the $T$-matrix plus LO pQCD scattering off gluons) are implemented into Langevin simulations of $c$ and $b$ quarks in $\mathrm{Au}-\mathrm{Au}$ collisions at RHIC using an isentropically expanding QGP fireball [16]. The latter has been constructed to resemble hydrodynamic models, with a bulk elliptic flow of $\sim 5.5 \%$ and initial temperature of $T_{0}=340 \mathrm{MeV}$ in semicentral collisions (when using LO pQCD interactions, the resulting HQ spectra are in good agreement with Langevin simulations in an explicit hydrodynamic background [11]). To compare to experimental $e^{ \pm}$spectra, $c$ and $b$ quarks are hadronized at the end of the QGPhadron gas mixed phase within the coalescence model of Ref. [33] (supplemented by $\delta$-function fragmentation and $D$ - and $B$-meson decays). Note that the resonance correlations in the $T$-matrix naturally merge into a quarkcoalescence description toward $T_{c}$ [34]. Our calculations for the $e^{ \pm}$nuclear modification factor, $R_{\mathrm{AA}}$ (defined as the ratio of the spectrum in $\mathrm{Au}-\mathrm{Au}$ collisions to the one in $p-p$ scaled by the number of binary $N-N$ collisions), and elliptic flow coefficient, $v_{2}$, show fair agreement with

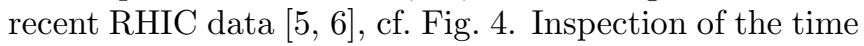
evolution of the $c$-quark distribution reveals that the suppression in the $p_{T}$ spectra (i.e., $R_{A A}$ ) is mostly built up in the early stages of the QGP, while most of the $v_{2}$ is developed at temperatures close to $T_{c}$. This feature is amplified by the temperature dependence of the transport coefficients in the $T$-matrix approach, and seems to 


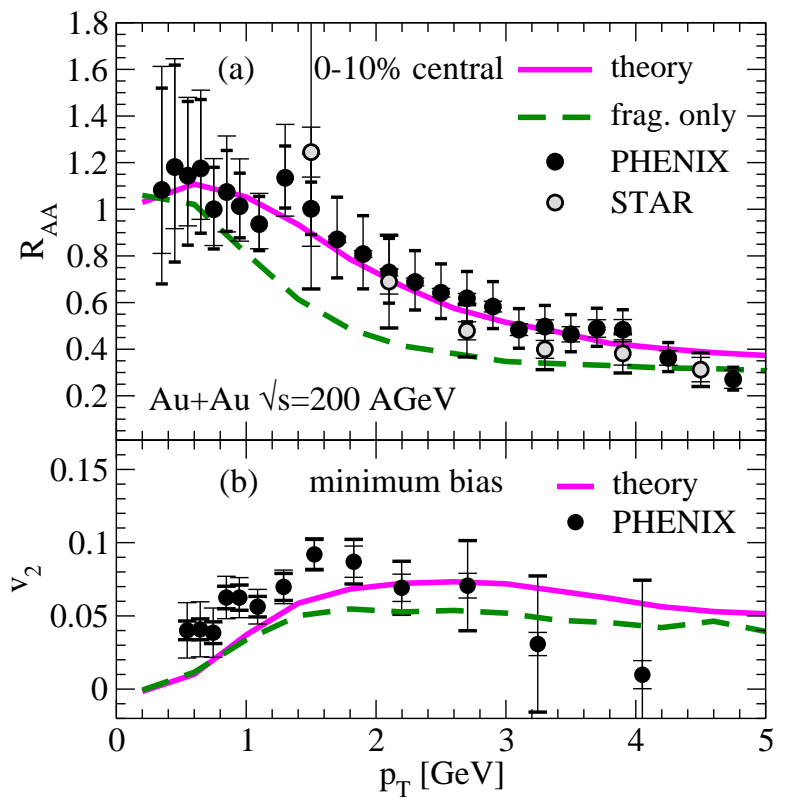

FIG. 4: Our results for the nuclear modification factor (upper panel) and elliptic flow (lower panel) of single electrons with/without (solid/dashed lines) quark coalescence in $\mathrm{Au}-\mathrm{Au}$ collisions compared to RHIC data [5, [6]. The estimated theoretical uncertainty due to different extractions of the lQCD based heavy-light quark potentials is up to $\sim 30 \%$ [29].

be favored by the $e^{ \pm}$data (coalescence with light quarks further contributes to the increase in both $v_{2}$ and $R_{A A}$ ).
In summary, we have calculated HQ selfenergies and transport coefficients within a $T$-matrix approach for heavy-light quark interactions in the QGP using twobody potentials estimated from lattice QCD. HQ scattering turns out to be dominated by "prehadronic" mesonic and diquark channels which increase in strength when approaching $T_{c}$. These correlations substantially accelerate thermal relaxation times compared to $\mathrm{pQCD}$ and provide for a natural onset of the hadronization process (in such a scenario, nonperturbative $\mathrm{HQ}$ interactions with gluons are less relevant). When implemented into Langevin simulations at RHIC, reasonable agreement with the suppression and elliptic flow of $e^{ \pm}$spectra from HQ decays emerges. This is rather remarkable in view of the largely parameter-free calculation of the transport coefficients. Future work should aim at scrutinizing the uncertainties inherent in the potential approach at finite temperature and in the extraction of the potential from lattice QCD. Further insights could be obtained from direct lQCD computations of heavy-light quark correlation functions in the QGP. In addition, elastic HQ interactions, which parametrically dominate at low $p_{T}$, should be supplemented by radiative energy loss [13] which takes over at high $p_{T}$. Our present analysis suggests that a small HQ diffusion coefficient arises from a nonperturbative interaction strength in a strongly coupled QGP.

This work has been supported by a U.S. National Science Foundation CAREER Award under grant PHY0449489 (HvH, RR), and by the Ministerio de Educación y Ciencia under grant AYA 2005-08013-C03-02 (MM).
[1] E.V. Shuryak, Prog. Part. Nucl. Phys. 53, 273 (2004).

[2] P. F. Kolb, U. W. Heinz, arXiv:nucl-th/0305084.

[3] F. Karsch, J. Phys. G 34, S627 (2007).

[4] S. S. Adler et al. [PHENIX Collaboration], Phys. Rev. Lett. 96, 032301 (2006).

[5] B. I. Abelev et al. [STAR Collaboration], Phys. Rev. Lett. 98, 192301 (2007).

[6] A. Adare et al. [PHENIX Collaboration], Phys. Rev. Lett. 98, 172301 (2007).

[7] N. Armesto et al., Phys. Lett. B 637, 362 (2006).

[8] S. Wicks et al., Nucl. Phys. A 784, 426 (2007).

[9] S. Caron-Huot and G. D. Moore, Phys. Rev. Lett. 100, 052301 (2008).

[10] H. van Hees, R. Rapp, Phys. Rev. C 71, 034907 (2005).

[11] G. D. Moore, D. Teaney, Phys. Rev. C 71, 064904 (2005).

[12] X. Zhu et al., Phys. Lett. B 647, 366 (2007).

[13] I. Vitev, A. Adil, H. van Hees, J. Phys. G 34, S769 (2007).

[14] B. Svetitsky, Phys. Rev. D 37, 2484 (1988).

[15] B. Zhang, L.W. Chen, C.M. Ko, Phys. Rev. C 72, 024906 (2005).

[16] H. van Hees, V. Greco, R. Rapp, Phys. Rev. C 73, 034913 (2006).
[17] M. Mannarelli, R. Rapp, Phys. Rev. C 72, 064905 (2005).

[18] M. Avila, Phys. Rev. D 49, 309 (1994).

[19] S. Godfrey, N. Isgur, Phys. Rev. D 32, 189 (1985).

[20] O. Kaczmarek et al., Nucl. Phys. Proc. Suppl. 129, 560 (2004).

[21] E. V. Shuryak, I. Zahed, Phys. Rev. D 70, 054507 (2004).

[22] C. Y. Wong, Phys. Rev. C 72, 034906 (2005).

[23] D. Cabrera, R. Rapp, Phys. Rev. D 76, 114506 (2007).

[24] A. Nakamura, T. Saito, Phys. Lett. B 621, 171 (2005).

[25] M. Döring et al., Phys. Rev. D 75, 054504 (2007).

[26] O. Kaczmarek et al., Prog. Theor. Phys. Suppl. 153, 287 (2004).

[27] P. Petreczky, private communication (2004).

[28] P. Petreczky, K. Petrov, Phys. Rev. D 70, 054503 (2004).

[29] H. van Hees et al., in preparation.

[30] R.H. Thompson, Phys. Rev. D 1, 110 (1970).

[31] R. Blankenbecler, R. Sugar, Phys. Rev. 142, 1051 (1966).

[32] K. Abe et al. [Belle Collaboration], Phys. Rev. D 69, 112002 (2004).

[33] V. Greco, C.M. Ko, R. Rapp, Phys. Lett. B 595, 202 (2004).

[34] L. Ravagli, R. Rapp, Phys. Lett. B 655, 126 (2007). 